\title{
Dynamic Load Modulation of High Power Amplifiers with Varactor-Based Matching Networks
}

\author{
Ali Soltani Tehrani, Hossein Mashad Nemati, Haiying Cao, Thomas Eriksson, Christian Fager \\ GigaHertz Centre, Chalmers University of Technology \\ SE-41296, Goteborg, Sweden
}

\begin{abstract}
In this paper, the results of dynamic load modulation on a high power amplifier is shown with experiments. A simple static nonlinear model is used as an inverse model, and by dynamically controlling both the input signal to the power amplifier and the load impedance, high efficiency operation of the power amplifier is achieved. The modulated measurements show the feasibility of dynamic load modulation for practical high power, high frequency applications.

Index Terms-Efficiency, load modulation network, power amplifiers, tunable-matching networks.
\end{abstract}

\section{INTRODUCTION}

In order to fully utilize the limited bandwidth spectrum in modern wireless communication systems, variable envelope modulation schemes have been developed. These techniques have resulted in communication signals that often have a high peak-to-average ratio (PAR), which requires power amplifiers (PA) to operate with significant back-off to behave linearly. In consequence, even PAs with high peak efficiency will have a severe reduction in efficiency.

The power amplifier is a vital component in the transmitter architecture, and is normally the main power-consuming device. Many techniques have been proposed to increase the efficiency of PAs in back-off, and dynamic load modulation (DLM) and dynamic supply modulation (also known as envelope tracking) have shown to be promising. Compared to dynamic supply modulation, the matching network of a DLM architecture generally does not consume any power [1], while in envelope tracking schemes, the envelope amplifiers efficiency and bandwidth could also be an issue.

A general block diagram of a dynamic load modulation transmitter architecture is shown in Fig. 1.

In the literature, a variety of configurations have been used for the tunable matching network to achieve efficiency improvement, with varying results [2], [3]. A common disadvantage has been that for all architectures presented, peak power levels and frequency of operation have been quite low. Recently in [4] a design for a load modulation architecture for high power amplifiers was presented. This design proposed a modular approach for the DLM architecture, separating the design of the PA and the tunable matching network.

In all these works, the experiments were done using static measurements and hence only provided static tuning. In practical modulators, static tuning is no longer possible, and the architecture has to be tested with respect to dynamic tuning of

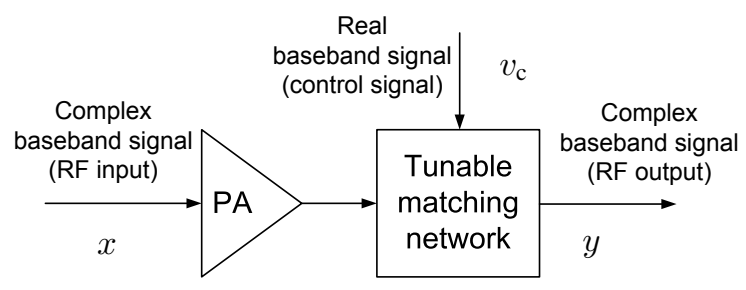

Fig. 1. General transmitter architecture for dynamic load modulation.

the varactors. This requires the design of the input signals to the PA and matching network such that the output signal has low distortion while the PA maintains high back-off efficiency.

In this work, we construct a simple inverse model for the architecture, design the input signals for the PA and matching network, and present modulated measurement results. This work shows the feasibility of the architecture with respect to dynamic tuning. To the authors knowledge, the first modulated measurements results for DLM on high power amplifiers is presented in this work.

\section{ARChitecture BACKGROUND}

From [4], a general procedure for designing a DLM network for a PA can be summarized as follows:

- Characterize the PA with load-pull measurements with a LSNA and find $P_{\text {out }}$, PAE vs $P_{i n}, \varphi_{i n}$, load impedance

- Design a varactor-based matching network (VMN) based on these measurements

- Design the required control functions for the PA and the VMN that result in the desired output signal using the static measurements

- Design the input signal for the PA and the VMN network with the control functions

In [5] it was shown that the maximum efficiency obtainable by controlling both input power and load impedance for a switched mode class-E $7 \mathrm{~W} 1 \mathrm{GHz}$ LDMOS PA [6] can theoretically be improved by up to 20 percentage units in backoff operations.

From the load-pull measurements the relationship between the output signal $y$, and the input amplitude, control voltage and phase can be constructed as following:

$$
y=f_{A}\left(|x|, v_{\mathrm{c}}\right) e^{-i f_{\varphi}\left(|x|, v_{\mathrm{c}}, L x\right)}
$$


where the RF input signal $x$ is assumed to consist of a timevarying amplitude $|x|$ and phase $\angle x$, and the baseband control voltage signal to consist of only an amplitude $v_{\mathrm{c}}$.

It is obvious that many combinations of the input signal and control voltage exist that provide the same output. In order to find the optimum, one has to constrain the configurations. One important constraint is that the combination should achieve maximum efficiency, which was used in this work.

In [4], a varactor-based tunable matching network was designed for the DLM architecture. The corresponding circuit configuration is shown in Fig. 2. The static performance of this

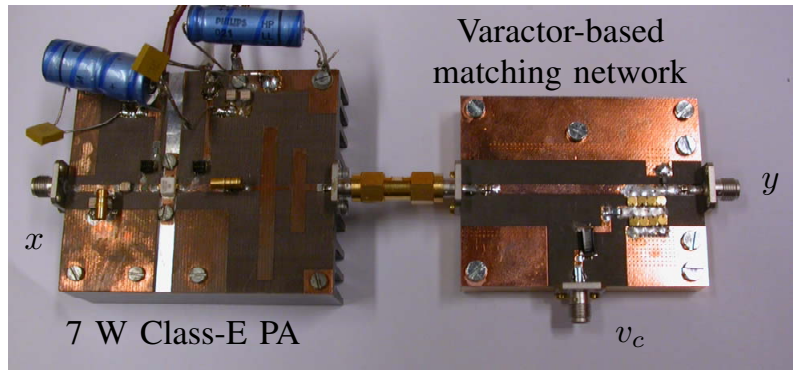

Fig. 2. The load modulated PA architecture.

architecture is shown in Fig. 3, where the optimum impedance locus is based on the load-pull measurements. It can be noticed that the achieved efficiency improvement is not the maximum efficiency due to the losses and mismatch associated with the practical implementation of the tunable network, but still is around $10 \%$ higher than the normal PA with $50 \Omega$ termination.

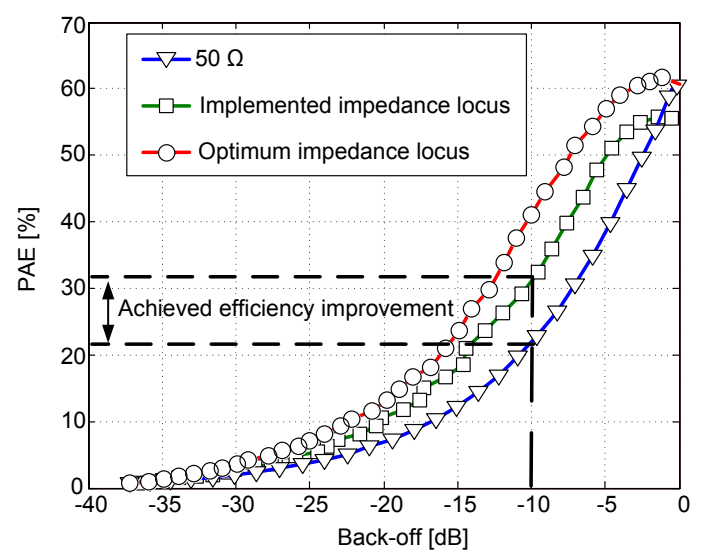

Fig. 3. Static performance of DLM architecture.

\section{INVERSE MODELING}

After designing the VMN, it is necessary to construct the input signals for the DLM architecture such that the output signal has low distortion while maintaining high efficiency for the PA in back-off. Therefore, inverse modeling of this architecture is necessary.

Due to the dual-input nature of the DLM architecture, design of the appropriate PA input signal and the baseband control voltage for the matching network is challenging. A general model for the architecture is shown in Fig. 4. In this work, a simple static nonlinear model is used.

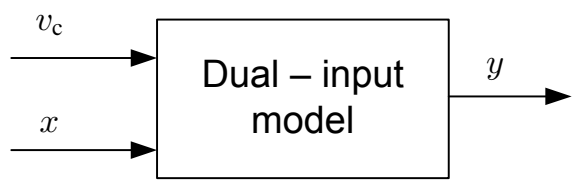

Fig. 4. General model for the DLM architecture.

\section{A. Input design}

In order to obtain the desired input, the first step is to measure the PA for different control voltage settings with varying drive levels. The optimum $v_{c}$ values that result in high PAE are then recorded. Once these optimum load impedances are found - if a one-to-one relationship between each optimum load impedance is assumed - they can be used to construct the optimum output-input amplitude, phase and control voltage functions.

$$
\begin{array}{rc}
|x|= & f_{A_{\mathrm{opt}}}(|y|) \\
\angle x= & f_{\varphi_{\mathrm{opt}}}(|y|, \angle y) \\
v_{\mathrm{c}} & =f_{Z_{\mathrm{opt}}}(|y|)
\end{array}
$$

For a desired complex output signal $y$, the complex input $x$ and the baseband input $v_{c}$ that result in the highest efficiency is constructed. This procedure results in a quasi-static inverse model of the architecture. The input-output relations obtained can be shown in Fig. 5 - 6 .

The optimum phase difference for the architecture with the varactor-based matching network (VMN) and without is shown in Fig. 6. It is interesting to note that the PA + VMN combination actually results in less AM/PM than the PA itself. This can be understood since the purpose of the VMN is actually to cancel the reactive effects in the device and restore a pure resistive load to the intrinsic device source.

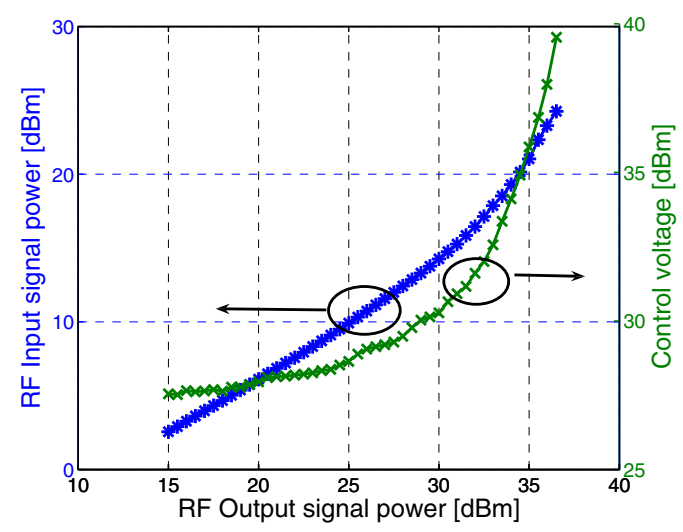

Fig. 5. Output-input power relations for optimum PAE performance.

With Fig. 5 and Fig. 6, a one-to-one relation for the output signal and input signals is available and it is now possible to 


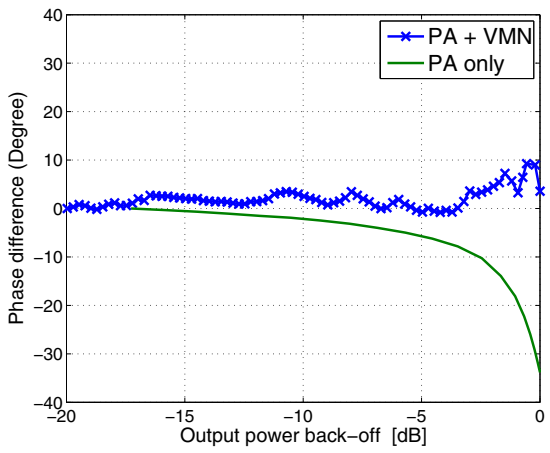

Fig. 6. Insertion phase difference of the power amplifier without the tunable network and for the PA with VMN.

construct the input signals such that the combination of the input and the control voltage yields high efficiency.

\section{B. Theoretical prediction values with modulated data}

In order to operate the DLM architecture as a practical transmitter, it is necessary to test the setup with modulated data. Before testing the setup and in order to prevent any major problems some theoretical prediction values have to be done.

The data that the PA is tested with is WCDMA data with a PAPR of $11.3 \mathrm{~dB}$. A polynomial fitting is done with the static measurements to obtain smooth functions for the modulated input signal and the control voltage for the VMN. These models were also used to extrapolate below the minimum $P_{\text {in }}$ used in the static measurements. Fig. 7 shows the PAE for the PA only, the PA+VMN with the efficiency optimized controlling scheme described above, and the modulated data's distribution. The PAE averaged over the pdf of the signal for

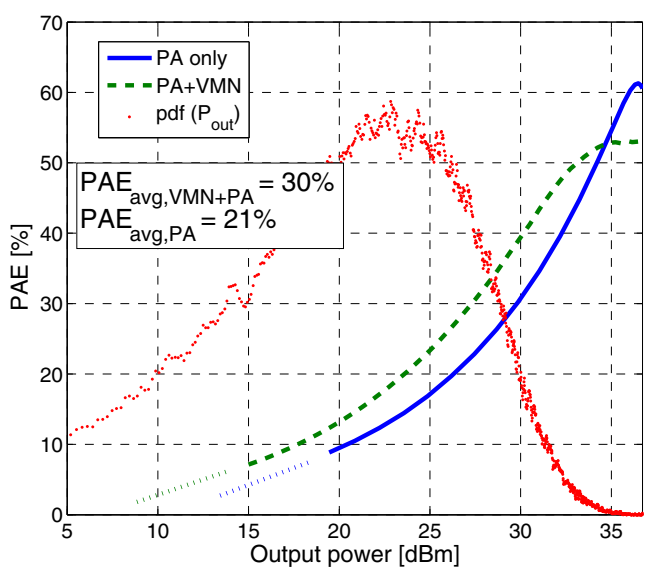

Fig. 7. Theoretical efficiency for the VMN network vs fixed $50 \Omega$ load impedance.

the PA without the varactor-based matching network is $21 \%$, while the PAE with the VMN is around $30 \%$. The respective drain efficiency is $22 \%$ and $31 \%$. With the use of the matching network, even though the peak output power PAE is lower, the overall predicted efficiency improvement is $9 \%$.

\section{Modulated Measurements}

In this section, the input signals obtained by the efficiency optimized functions is applied with the WCDMA signal and given to the PA and the varactor-based matching network. Due to severe bandwidth limitations of the PA used, it was not possible to perform the measurements at the full WCDMA bandwidth. The signal bandwidth was therefore down-scaled with a factor of 10 during the measurements presented.

\section{A. Measurement setup and issues}

The measurement setup used in this experiment is shown in Fig. 8. After finding the optimum functions, the input



Fig. 8. Measurement setup used in the experiment.

signals are calculated and the complex baseband input signal is uploaded to an Agilent E4438C vector signal generator that acts as a modulator, and the control voltage for the VMN is uploaded to a Tabor Electronics, WW2572A arbitrary waveform generator (AWG). An Agilent 54845A digital storage oscilloscope (DSO) is used as a vector signal analyzer. The data is then downloaded to the computer where down conversion to baseband and time alignment are done. All devices are connected by GPIB and triggered in synchronization.

In order to achieve the necessary dynamic range on the VMN, a voltage swing of $6-27 \mathrm{~V}$ on the varactors was needed. The AWG used in this experiment could not provide such voltages, so an op-amp circuit was constructed to amplify the control voltage signal to the necessary level.

An important issue for this experiment, is the time alignment of the input signal to the matching network, and the control voltage. In order to achieve high efficiency, it is necessary that when the magnitude of the PA output is high, the voltage applied to the matching network is high simultaneously. Even slight time alignments may result in degradation in the overall efficiency and particularly in linearity. In this work the time alignment was done manually, and with more advanced schemes better results are obtainable.

\section{B. Results}

The magnitude of the complex input signal and the control voltage is shown in Fig. 9. From this figure, it can be observed that the baseband control voltage correctly follows the input. The measured input- and output spectra is shown in Fig. 10. The spectrum for the control voltage is also shown in Fig. 11. 


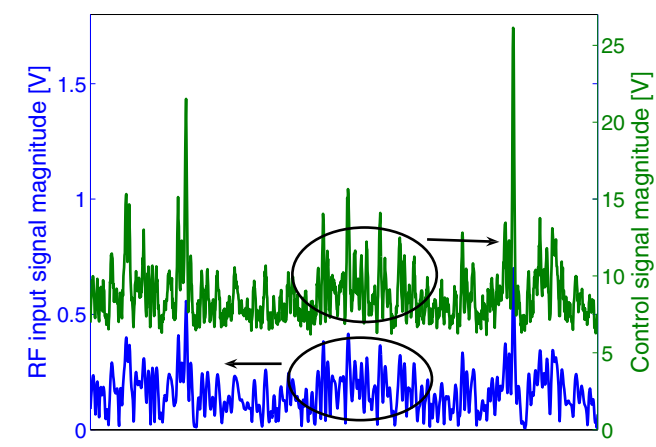

Fig. 9. Magnitude of the RF input signal and the baseband control signal.

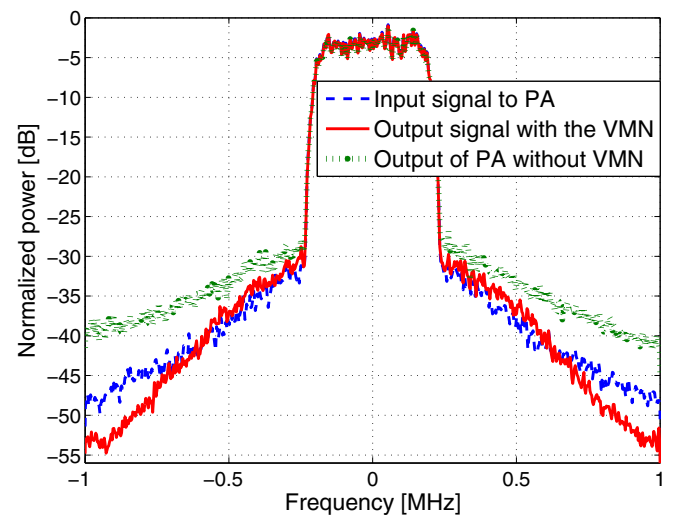

Fig. 10. Signal spectrum of the RF input signal to the PA and output signal of the VMN.

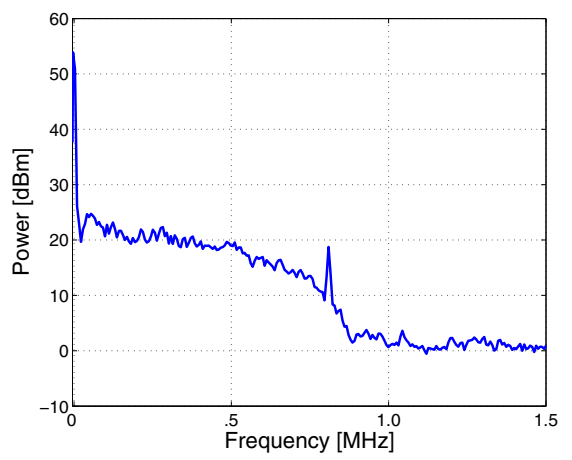

Fig. 11. Signal spectrum of the baseband input signal to the VMN.

It can be noticed from these figures that the signal bandwidths are of practical values. The bandwidth of the control signal which contains $95 \%$ of the power is $1.1 \mathrm{MHz}$.

These signals are uploaded to the devices and the modulated measurements are done on the PA alone and on the DLM architecture, resulting in output powers of 0.38 and $0.44 \mathrm{~W}$ respectively. The resulting efficiency is summarized in Table I. It can be noticed that the measured results agree fairly well
TABLE I

EFFICIENCY RESULTS FOR THE MODULATED MEASUREMENTS

\begin{tabular}{c|c} 
Architecture & Average PAE \% \\
\hline \hline PA from static estimation & 21 \\
PA + VMN from static estimation & 30 \\
PA with measurements & 19 \\
PA + VMN with measurements & 31
\end{tabular}

with the predictions.

With the help of the VMN, the PAE has around 12\% improvement. It can be also be noticed in Fig. 10 that the output signal has less spectral regrowth compared to the PA without any matching network. With more advanced modeling, the linearity of the architecture can be further enhanced.

The dual-input nature of such an architecture allows one extra degree of freedom for the PA, which can result in better efficiency, better linearity or a combination of both. To the authors knowledge, these are the first modulated measurement results done on a high power, high frequency PA with the help of dynamic load modulation.

\section{Conclusions}

The feasibility of dynamic load modulation with varactor based networks for high power amplifiers was shown in this work. With a simple quasi-static inverse model it was possible to construct the required input signals. The results shows significant improvement in back-off efficiency for the architecture compared to the normal PA configuration with a fixed $50 \Omega$ load impedance can be achieved.

The dual-input nature of the DLM architecture has great modeling challenges, but also gives great possibilities for further improving the linearity and efficiency.

\section{ACKNOWLEDGEMENT}

This research has been carried out in the GigaHertz centre in a joint research project financed by the Swedish Governmental Agency of Innovation Systems (VINNOVA), Chalmers University of Technology, ComHeat Microwave, Ericsson AB, Infineon Technologies, NXP Semiconductors and Saab AB.

\section{REFERENCES}

[1] F. H. Raab, "High-efficiency linear amplification by dynamic load modulation," in Proc. IEEE MTT-S Int. Micr. Symp. Dig., vol. 3, 2003, pp. $1717-1720$.

[2] W. C. E. Neo, Y. Lin, X. D. Liu, L. C. N. De Vreede, L. E. Larson, M. Spirito, M. J. Pelk, K. Buisman, A. Akhnoukh, A. De Graauw, and L. K. Nanver, "Adaptive multi-band multi-mode power amplifier using integrated varactor-based tunable matching networks," Solid-State Circuits, IEEE Journal of, vol. 41, no. 9, pp. 2166-2176, 2006.

[3] J.-S. Fu and A. Mortazawi, "A tunable matching network for power amplifier efficiency enhancement and distortion reduction," in Proc. IEEE MTT-S Int. Micr. Symp. Dig., 2008, pp. 1151-1154.

[4] H. M. Nemati, C. Fager, U. Gustavsson, R. Jos, and H. Zirath, "Design of varactor-based tunable matching networks for dynamic load modulation of high power amplifiers," Submitted to IEEE Trans. Micro. Theory Tech.

[5] H. M. Nemati, C. Fager, U. Gustavsson, and H. Zirath, "An efficiency optimized controlling scheme for dynamic load modulation of power amplifiers," in Proc. European Microwave . Conf. $38^{\text {th }}$, Oct. 2008, pp. 583-586.

[6] A. Adahl and H. Zirath, "An $1 \mathrm{GHz}$ class E LDMOS power amplifier," in Proc. European Microwave Conf. 33 ${ }^{\text {rd }}$, 2003, pp. 285-288. 